\title{
Prevalência de desvio de septo nasal em crianças e adolescentes de um hospital terciário
}

\section{Prevalence of nasal septum deviation in children and adolescents of a tertiary hospital}

\begin{abstract}
Willian Silva Lopes ${ }^{1}$. Camila Alencar Moreira ${ }^{2}$. Viviane Carvalho da Silva ${ }^{3}$. Marcos Rabelo de Freitas ${ }^{4}$.
1 Residente de Otorrinolaringologia, Hospital Universitário Walter Cantídio (HUWC), Fortaleza, Ceará, Brasil. 2 Otorrinolaringologista, Doutora em Microbiologia Médica pela Universidade Federal do Ceará (UFC), Fortaleza, Ceará, Brasil. 3 Mestrado em Saúde Pública, Otorrinolaringologista, Médica assistente e Coordenadora da Residência Médica de Otorrinolaringologia, Hospital Universitário Walter Cantídio (HUWC), Universidade Federal do Ceará (UFC), Fortaleza, Ceará, Brasil. 4 Doutor em Cirurgia, Otorrinolaringologista, Departamento de Cirurgia, Universidade Federal do Ceará (UFC), Fortaleza, Ceará, Brasil.
\end{abstract}

\section{RESUMO}

Introdução: Os desvios de septo nasal (DSN) podem ser assintomáticos ou apresentar obstrução nasal que interfere na qualidade de vida e no crescimento facial. Tem etiologia multifatorial. Objetivo: Avaliar a prevalência total e de determinados tipos de DSN em crianças e adolescentes, relacionando-os a sexo e idade. Método: pacientes de ambulatório de Otorrinopediatria de um hospital terciário, com idade até 16 anos completos, avaliadas através de anamnese em questionário padrão e exame físico, para classificação de DSN segundo Mladina. Identificado a presença ou não de deformidade, relacionando-a a sexo, faixa etária e tipo de desvio. Resultados: 497 crianças avaliadas, nas quais 36,8\% havia DSN, sendo 37,8\% no sexo masculino e $35,6 \%$ no sexo feminino. Identificou-se prevalência de 24,3\% em menores de 6 anos de idade, $41,5 \%$ em crianças entre $6-11$ anos e 59,6\% em maiores de 11 anos. A prevalência por tipo foi de $36,5 \%$ no tipo $1,13,1 \%$ no tipo $2,12,5 \%$ no tipo $3,4,9 \%$ no tipo $4,14,7 \%$ no tipo 5, 13,1\% no tipo 6 e 4,9\% no tipo 7. Conclusão: A prevalência de DSN em crianças e adolescentes até 16 anos foi de 36,8\%, aumentando com a idade. Não houve diferença quanto ao sexo. Os mais prevalentes foram os tipos 1 e 5.

Palavras-chave: Septo nasal. Criança. Deformidades adquiridas nasais. Prevalência.

\section{ABSTRACT}

Introduction: The nasal septum deviation (NSD) may be asymptomatic, or present nasal obstruction that changes quality of life and facial growth, sometimes with aesthetic influence. It has a multifactorial etiology. Objective: to evaluate the total and specific types of NSD prevalence in children, and to relate them to sex and age. Method: children of Otorhinolaryngology clinic from a tertiary hospital, aged less than 17 years, were evaluated with physical examination to classify NSD according to Mladina. Presence or absence of deformity were evaluated, relating to sex, age and type of deviation. Results: 497 children were evaluated, identifying NSD in $36.8 \%$, with $37,8 \%$ in males and $35.6 \%$ in females. In age groups, the prevalence was $24.3 \%$ in children under 6 years of age, $41.5 \%$ aged $6-11$ years and $59.6 \%$ in those over 11 years old. The prevalence of each type of NSD was $36.5 \%$ in type $1,13.1 \%$ in type $2,12.5 \%$ in type $3,4.9 \%$ in type $4,14.7 \%$ in type $5,13.1 \%$ in type 6 and $4.9 \%$ in type 7 . Conclusion: The prevalence of NSD in children was $36.8 \%$, increased with age. There was no difference between the sexes. The NSD type 1 and 5 were the most prevalent.

Keywords: Nasal septum. Child. Nose deformities. Prevalence.

Autor correspondente: Willian da Silva Lopes, Rua Capitão Francisco Pedro, 1290, Rodolfo Teófilo, Fortaleza, Ceará. CEP: 60430-372. Telefone: +55 85 3366-8616. E-mail: willdsilopes@hotmail.com

Conflito de interesses: Não há qualquer conflito de interesses por parte de qualquer um dos autores.

Recebido em: 19 Abr 2017; Revisado em: 24 Set 2017; Aceito em: 18 Out 2017. 


\section{INTRODUÇÃO}

O septo nasal é formado por um componente ósseo, definido pelo vômer, lâmina perpendicular do etmoide, crista septal do maxilar e do palatino e, sobre estes, um componente cartilaginoso denominado cartilagem quadrangular. ${ }^{1} \mathrm{~A}$ área septal onde há a fusão da cartilagem quadrangular, cartilagens laterais superiores, ossos próprios nasais e lâmina perpendicular do etmoide é conhecida como área $\mathrm{K}$ e é responsável pela sustentação da pirâmide nasal. $\mathrm{O}$ conhecimento morfofisiológico dessa região tem permitido mudanças no manejo dos desvios de septo nasal (DSN), sendo observadas intervenções em idade cada vez mais precoce ${ }^{2}$ com a justificativa de que o restabelecimento da respiração nasal levaria à normalização do desenvolvimento da criança como um todo, principalmente no terço médio da sua face. ${ }^{3}$ Esse desenvolvimento permite a execução de funções progressivamente mais complexas e perfeitas, como respirar, mastigar e deglutir. ${ }^{4}$

Muitos desvios septais estão presentes ao nascimento, evidenciando etiologias que abrangem desde a hereditariedade ${ }^{5}$ a alterações durante o período gestacional ${ }^{6}$ e periparto. $^{7}$ Modificações na dinâmica do crescimento facial por influência de fatores ocorridos durante a infância, ${ }^{6}$ como a síndrome do respirador oral, implicariam em deformações septais. Mas ainda não se sabe ao certo se esses fatores seriam causa ou consequência das alterações no crescimento facial. Os traumas faciais são comprovadamente relevantes fatores causais. ${ }^{8,9}$

Os DSN podem ter diversas manifestações clínicas, variando desde pacientes assintomáticos, até obstrução nasal que interfere na qualidade de vida e no crescimento da face. ${ }^{10}$ Algumas vezes, ainda, comprometem a estética nasal com consequentes implicações psicológicas.

A decisão da melhor época de intervenção cirúrgica em crianças é controversa, devendo-se avaliar o impacto que os DSN promovem no desenvolvimento facial e manter preservado o crescimento septal proporcional ao crescimento facial desses pacientes. ${ }^{11}$

A prevalência dos DSN em crianças tem sido relatada por alguns autores. Em recentes estudos envolvendo a faixa etária pediátrica, Mladina e Subaric (2002) estudaram crianças e adolescentes de 2-22 anos na Croácia, utilizando a classificação de Mladina para localizar o nível do DSN. ${ }^{12}$ Em 2003, Yildirim e Okur fizeram o mesmo com crianças de 4-16 anos na Turquia, ${ }^{10}$ e em 2004 Zielnik-Jurkiewicz e Olszewska-Sosinska avaliaram crianças de 3-17 anos na Polônia. ${ }^{13}$

No Brasil, há poucos estudos de prevalência de DSN envolvendo crianças. ${ }^{14,15}$ Nenhum envolve exclusivamente a faixa etária pediátrica. Novos estudos de prevalência de DSN são, portanto, necessários para avaliações epidemiológicas mais significativas em crianças.

Assim, o presente estudo tem como objetivo avaliar a prevalência dos desvios de septos nasais em crianças e adolescentes acompanhadas pelo serviço de Otorrinolaringologia de um hospital terciário, identificando os tipos de DSN de acordo com a classificação de Mladina e analisando a distribuição desses conforme idade e sexo.

\section{MÉTODOS}

Foram avaliados pacientes atendidos em ambulatório de Otorrinopediatria de um hospital terciário, onde há otorrinolaringologistas especializados nesse atendimento. Foram incluídas crianças e adolescentes com idade até 16 anos completos, de ambos os sexos, atendidas entre junho e dezembro de 2007 e entre setembro e novembro de 2015, independente de terem ou não queixas nasais. Por tratar-se de dois períodos totalmente distintos, nenhuma criança foi reavaliada. Participantes sindrômicas foram excluídas da pesquisa.

O estudo foi aprovado pelo Comitê de Ética em Pesquisa da instituição sob parecer de número 1.357.537. Todos os responsáveis pelos pacientes foram informados sobre a pesquisa e convidados a participar, aceitando espontaneamente e assinando consentimento esclarecido.

Um questionário foi aplicado, incluindo perguntas sobre existência de trauma nasal, alergia, sintomas presentes e tratamento otorrinolaringológico anterior. Dados do exame físico (rinoscopia anterior com uso de espéculo nasal), classificando os DSN quanto ao tipo e a gravidade foram também registrados. Não se fez uso de avaliação por endoscopia nasal ou exame complementar por imagem.

Os critérios de inclusão foram crianças abaixo de 17 anos, independente das queixas.

Foi utilizada a classificação de Mladina (Quadro 1 e Figura 1) para os DSN através de rinoscopia anterior sem uso de vasoconstrictores.

Quadro 1. Classificação de DSN segundo Mladina.

\begin{tabular}{|l|l|}
\hline $\begin{array}{l}\text { Tipo de desvio } \\
\text { de septo nasal }\end{array}$ & Classificação de Mladina \\
\hline Tipo 1 & $\begin{array}{l}\text { Crista vertical na área da válvula nasal não } \\
\text { atingindo dorso nasal. }\end{array}$ \\
\hline Tipo 2 & $\begin{array}{l}\text { Crista vertical unilateral em contato com a } \\
\text { válvula nasal. }\end{array}$ \\
\hline Tipo 3 & Crista vertical mais profunda. \\
\hline Tipo 4 & $\begin{array}{l}\text { Deformidade em "forma de S" que alcança dorso } \\
\text { nasal. }\end{array}$ \\
\hline Tipo 5 & $\begin{array}{l}\text { Quase plano de um lado e um esporão horizontal } \\
\text { basal no outro lado. }\end{array}$ \\
\hline
\end{tabular}

Continua. 
Conclusão.

Quadro 1. Classificação de DSN segundo Mladina.

\begin{tabular}{|l|l|}
\hline $\begin{array}{l}\text { Tipo de desvio } \\
\text { de septo nasal }\end{array}$ & Classificação de Mladina \\
\hline Tipo 6 & $\begin{array}{l}\text { Chanfradura horizontal profunda no segmento } \\
\text { basal septal anterior de um lado e cume formado a } \\
\text { partir de uma asa de osso intermaxilar contralateral. }\end{array}$ \\
\hline Tipo 7 & $\begin{array}{l}\text { Multipicidade de desvios (septo "amassado"), } \\
\text { combinação dos anteriores. }\end{array}$ \\
\hline
\end{tabular}

Figura 1. Classificação DSN segundo Mladina.

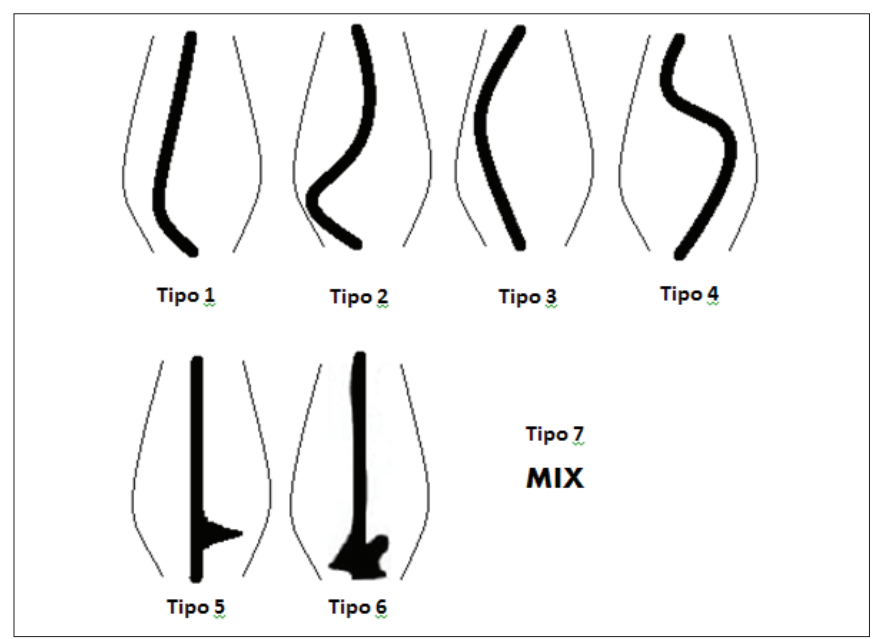

Adaptado de: Mladina e Subaric. ${ }^{12}$

Tais crianças foram cadastradas em banco de dados para organização dos dados coletados (Microsoft Excel Software) e análises estatísticas feitas por programa Graphpad prism 5. As diferenças entre os tipos de deformidades do septo nasal e entre sexos e faixas etárias, as quais foram divididas em três grupos ( $<6$ anos, entre 6 e 11 anos e $>11$ anos), foram testadas por $\chi 2$-teste para amostras independentes e teste de Fischer.

\section{RESULTADOS}

Foram avaliados 351 pacientes durante o ano de 2007 e 146 em 2015, fazendo um total de 497 crianças participantes do estudo.

A prevalência de DSN foi de 36,8\%, identificando-se $37,8 \%$ e $35,6 \%$ de prevalência nos sexos masculino e feminino respectivamente. Contudo, não houve diferença estatisticamente significante $(\chi 2, \mathrm{p}>0,05)$.
Quanto à prevalência do DSN por faixa etária, identificou-se $24,3 \%$ em $<6$ anos de idade, $41,5 \%$ entre $6-11$ anos e $59,6 \%$ em $>11$ anos (Gráfico 1), observando-se um aumento significativo da prevalência diretamente proporcional à idade $(\chi 2, \mathrm{p}<0,0001)$.

Avaliando-se os pacientes que apresentam DSN quanto à classificação de Mladina, pôde-se estabelecer a prevalência de cada tipo de desvio (1-7). A prevalência foi de $36,6 \%$ para tipo 1 , $13,1 \%$ para tipo $2,12,5 \%$ para tipo $3,4,9 \%$ para tipo $4,14,7 \%$ para tipo 5, 13,1\% para tipo 6 e 4,9\% para tipo 7 (Gráfico 2).

A distribuição de cada tipo de desvio por faixa etária está apresentada na Tabela 1 e por sexo na Tabela 2 .

Gráfico 1. Desvio de septo nasal por faixa etária $\left(\chi 2,{ }^{*} p<0,0001\right)$.

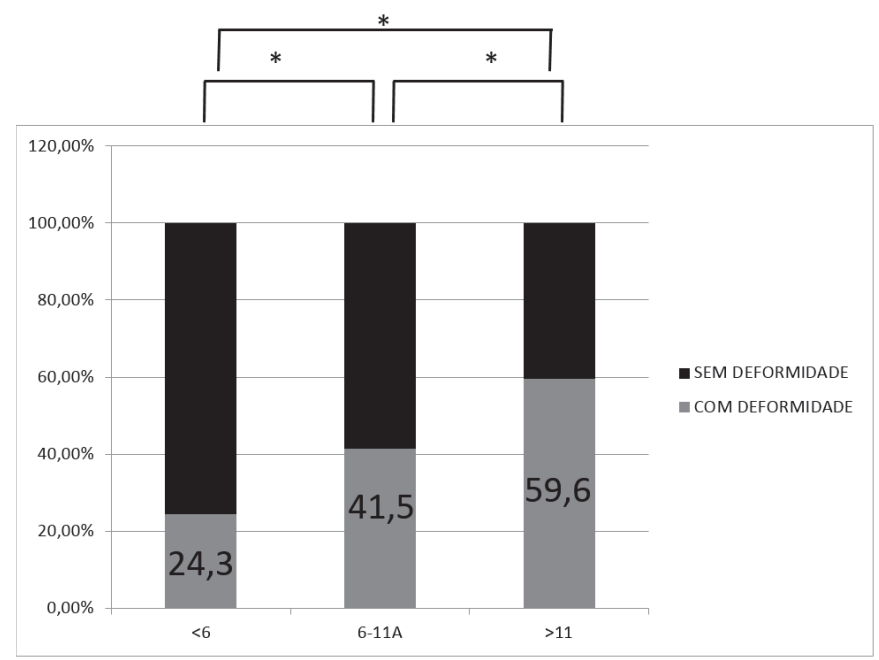

Gráfico 2. Prevalência dos tipos de desvio de septo.

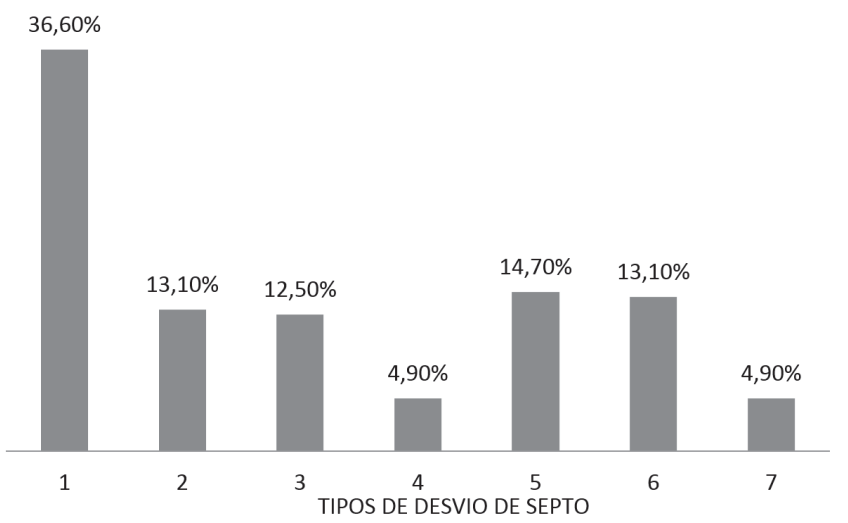

Tabela 1. Tipo de deformidade do septo nasal de acordo com a faixa etária.

\begin{tabular}{ccccccccc}
\hline Faixa etária & $\begin{array}{c}1 \\
\mathrm{n}(\%)\end{array}$ & $\begin{array}{c}2 \\
\mathrm{n}(\%)\end{array}$ & $\begin{array}{c}3 \\
\mathrm{n}(\%)\end{array}$ & $\begin{array}{c}4 \\
\mathrm{n}(\%)\end{array}$ & $\begin{array}{c}5 \\
\mathrm{n}(\%)\end{array}$ & $\begin{array}{c}6 \\
\mathrm{n}(\%)\end{array}$ & $\begin{array}{c}7 \\
\mathrm{n}(\%)\end{array}$ & Total \\
\hline$<6$ & $20(44,4)$ & $5(11,1)$ & $8(17,8)$ & $3(6,7)$ & $6(13,3)$ & $3(6,7)$ & $0(0)$ & $45(100)$ \\
$6-11$ & $40(36,4)$ & $13(11,8)$ & $10(9,1)$ & $5(4,5)$ & $18(16,4)$ & $16(14,5)$ & $8(7,3)$ & $110(100)$ \\
$>11$ & $7(25)$ & $6(21,4)$ & $5(17,8)$ & $1(3,6)$ & $3(10,7)$ & $5(17,8)$ & $1(3,6)$ & $28(100)$ \\
\hline
\end{tabular}

n: número; \%: percentagem 
Tabela 2. Tipo de deformidade do septo nasal de acordo com sexo.

\begin{tabular}{crrrrrrrr}
\hline Sexo & \multicolumn{1}{c}{$\begin{array}{c}2 \\
\mathrm{n}(\%)\end{array}$} & $\mathrm{n}(\%)$ & $\mathrm{n}(\%)$ & $\mathrm{n}(\%)$ & $\begin{array}{c}5 \\
\mathrm{n}(\%)\end{array}$ & $\begin{array}{c}6 \\
\mathrm{n}(\%)\end{array}$ & $\begin{array}{c}7 \\
\mathrm{n}(\%)\end{array}$ & Total \\
\hline Masculino & $35(52,2)$ & $15(62,5)$ & $14(60,9)$ & $3(33,3)$ & $17(63)$ & $14(58,3)$ & $6(66,7)$ & $104(56,8)$ \\
Feminino & $32(47,8)$ & $9(37,5)$ & $9(39,1)$ & $6(66,7)$ & $10(37)$ & $10(41,7)$ & $3(33,3)$ & $79(43,2)$ \\
Total & 67 & 24 & 23 & 9 & 27 & 24 & 9 & $183(100)$ \\
\hline
\end{tabular}

n: número; \%: percentagem

\section{DISCUSSÃO}

O desenvolvimento do septo nasal é influenciado por múltiplos fatores, sejam eles genéticos ou ambientais.

DSN foi observado em $37,2 \%$ das crianças entre 0 e 16 anos acompanhadas em serviço de Otorrinolaringologia de um hospital terciário brasileiro neste estudo. Prevalência de 33,68\% das crianças entre 3-17 anos em Varsóvia - Polônia, ${ }^{13}$ $28,9 \%$ entre 2-22 anos em Zagreb - Croácia ${ }^{12}$ e 34,9\% entre 4-16 anos em Kahramanmaras - Turquia ${ }^{10}$ foi identificada em estudos anteriores. A classificação de Mladina ${ }^{12}$ e rinoscopia anterior sem vasoconstrictor foram utilizadas em todos os estudos, mas, apenas neste, a população avaliada foram aqueles acompanhados em serviço de um hospital terciário. Nos demais, a população alvo foi de crianças em idade escolar, fora do ambiente hospitalar. Mesmo com tal diferença de metodologia, as prevalências foram similares em todos os estudos.

Todos os estudos, incluindo este, identificaram o sexo masculino como mais prevalente na população com DSN, exceto o realizado em Zagreb onde predominou sexo feminino $(52,1 \%){ }^{12}$ Apenas em Varsóvia a predominância masculina dos DSN foi estatisticamente significante $(59,4 \%) .{ }^{13}$ Neste estudo, apenas o DSN tipo 4 foi mais prevalente no sexo feminino. A maior prevalência de DSN no sexo masculino encontrado na maioria dos trabalhos pode refletir o fato de este sexo ser mais suscetível a traumas. ${ }^{12}$

Os estudos não são homogêneos na divisão de faixa etária, com variações nas idades limites da faixa intermediária. Todavia, em todos os estudos o terceiro grupo, que contém a faixa etária mais velha $(>11,12,13$ ou 14 anos, dependendo do estudo) apresentou maior prevalência percentual do DSN, com diferença estatística significante observada também em todos eles, o que sugere um papel dos fatores ambientais influenciando a formação do DSN ao longo do tempo. ${ }^{12}$

Quanto ao tipo de desvio de septo, Zielnik-Jurkiewicz e Olszewska-Sosinska ${ }^{13}$ e Yildirim e Okur ${ }^{10}$ identificaram como mais prevalente, em ordem decrescente, tipos 1, 5 e 3. Subaric

\section{REFERÊNCIAS}

1. Oliveira MG, Barros FJ. Osteologia. In: Oliveira MG. Anatomy manual of the head and neck. 4 ed. Porto Alegre: EDPUCRS; 2002. p. 17-26.

2. Piltcher O. Septoplasty in children: problem or solution? Braz J Otorhinolaryngol. 2013;79:408. e Mladina ${ }^{12}$ identificaram tipos 1, 2 e 5 como mais prevalentes. Por sua vez, este estudo identificou tipos 1, 5 e 6 como mais prevalentes. Assim, em todos os estudos o tipo 1 foi o DSN mais encontrado em todas as faixas etárias, com exceção do realizado em Varsóvia onde, no terceiro grupo etário (entre 14 e 17 anos), houve maior prevalência do DSN tipo $5 .{ }^{13}$

O achado de aumento de determinados tipos de DSN com o decréscimo de idade, como ocorrido em Zagreb ${ }^{12}$ e Varsóvia, ${ }^{13}$ não foi encontrado no presente estudo, não havendo relação da idade com tipo de desvio. Em Kahramanmaras, ${ }^{10}$ essa relação de aumento de DSN com decréscimo de idade é observada em todos os tipos, exceto nos tipos 3 e 4 .

Correspondendo os tipos 1, 2 e 6 a DSN anteriores e os tipos 3, 4 e 5 a posteriores, segundo Mladina, ${ }^{12}$ podemos inferir que neste estudo os desvios anteriores correspondem a $64,3 \%$ e os posteriores a $31,7 \%$, sendo, portanto, cerca de duas vezes mais frequentes. Essa relação entre desvios anteriores e posteriores também foi observada por Subaric e Mladina ${ }^{12}$ e Yildirim e Okur, ${ }^{10}$ mas com proporção cinco vezes mais frequente dos desvios anteriores no estudo em Zagreb. ${ }^{12}$ Tais estudos relacionam DSN anteriores a injúrias. ${ }^{13}$ Zielnik-Jurkiewicz e Olszewska-Sosinska ${ }^{13}$ tiveram relação inversa, com maior prevalência do DSN posterior.

A relação entre aumento da prevalência dos DSN posteriores em crianças do terceiro grupo etário nos três estudos prévios, o que sugere intenso crescimento nasomaxilar nessa faixa etária, ${ }^{13}$ não foi identificada no presente estudo, onde se viu manutenção da prevalência do DSN anterior sobre o posterior, com poucas variações entre as faixas etárias.

\section{CONCLUSÃO}

As deformidades septais apresentaram elevada prevalência em crianças deste estudo, acometendo mais de 1/3 dos pacientes avaliados. Houve um predomínio no sexo masculino e um aumento da prevalência nas idades maiores. As deformidades mais comuns de acordo com a classificação de Mladina foram as tipo 1 e 5. Neste grupo etário, as deformidades anteriores foram mais prevalentes que as posteriores.

3. Costa DB, Anselmo-Lima WT, Tamashiro E, Enoki C, Valera FC. The impact of Metzembaum septoplasty on nasal and facial growth in children. Braz J Otorhinolaryngol. 2013;79:454-9.

4. Maniglia JV, Molina FD, Maniglia LP, Maniglia CP. 
Septorhinoplasty in children. Braz J Otorhinolaryngol. 2002;68:320-3.

5. Mladina R, Subaric M. Are some septal deformities inherited? Type 6 revisited. Int J Pediatr Otorhinolaryngol. 2003;67:1291-4

6. Carpes LF, Fiori HH. Etiology and treatment of nasal septum deviation in newborn infants: literature review. Scientia Medica. 2008;18:92-7.

7. Nakao M, Belizza, VI. Incidence of deviation os nasal septum in newly born [Internet]. São Paulo: Moreira JR editora; 2000 [acesso em: 28 mai 2014]. Disponível em: http://www.moreirajr.com.br/ revistas.asp? fase $=$ r003\&id_materia $=1378$

8. Pedroni PU, Rispoli DZ, Fonseca VR, Medeiros AI, Marcelino TF, Moller LG, et al. Septoplasty in children. Braz J Otorhinolaryngol. 2008;74:1-7.

9. Dispenza F, Saraniti C, Sciandra D, Kulamarva G, Dispenza C. Management of naso-septal deformity in childhood: long-term results. Auris Nasus Larynx. 2009;36:665-70.
10. Yildirim I, Okur E. The prevalence of nasal septal deviation in children from Kahramanmaras, Turkey. Int J Pediatr Otorhinolaryngol. 2003;67:1203-6.

11. Pereira PS, Kajiwara JK, Grellet M. Morphologic study of the development of the human nose quadrilateral cartilage and implications in the septoplastic surgery. Braz J Otorhinolaryngol. 2002;68:209-17.

12. Mladina R, Subaric M. Nasal septum deformities in children and adolescents: a cross sectional study of children from Zagreb, Croatia. Int J Pediatr Otorhinolaryngol. 2002;63:41-8.

13. Zielnik-Jurkiewicz B, Olszewska-Sosinska O. Nasal septum deformities in children and adolescents from Warsaw. Int J Pediatr Otorhinolaryngol. 2006;70:731-6.

14. Neves-Pinto R, Saraiva M. On the incidence of septal deformities according to Mladina's classification and some correlated aspects. Folha Med. 1993;106:73-7.

15. Oliveira AK, Junior EE, Santos LV, Bettega G, Mocellin M. Prevalence of deviated nasal septum in Curitiba, Brazil. Int Arch Otorhinolaryngol. 2005;9:288-92.

\section{Como citar:}

Lopes WS, Moreira CA, Silva VC, Freitas MR. Prevalência de desvio de septo nasal em crianças e adolescentes de um hospital terciário. Rev Med UFC. 2018 jul-set;58(3):25-29. 